Classification

Physics Abstracts

$61.12 \mathrm{D}-61.30 \mathrm{E}$

\title{
Internal structure of aggregates of two amphiphilic species in a lyotropic liquid crystal
}

\author{
S. Alpérine, Y. Hendrikx and J. Charvolin \\ Laboratoire de Physique des Solides $\left({ }^{+}\right)$, Bât. 510, Université de Paris-Sud, 91405 Orsay, France \\ and GRECO, Microemulsions (CNRS)
}

(Reçu le 27 juillet 1984, accepté le 6 novembre 1984)

\begin{abstract}
Résumé. - On étudie par diffusion de neutrons la structure interne d'un agrégat en forme de ruban dans une mésophase ordonnée à symétrie rectangulaire formée par deux amphiphiles, décyl sulfate de sodium et décanol, en présence d'eau. Les résultats expérimentaux montrent que les deux amphiphiles ne sont pas distribués de manière uniforme dans l'agrégat. Leur concentration relative varie localement selon la courbure interfaciale de l'agrégat. Une telle relation entre la concentration et la courbure peut être un point important pour comprendre la forme des agrégats et donc le polymorphisme des cristaux liquides lyotropes.
\end{abstract}

\begin{abstract}
The internal structure of a ribbon-like aggregate in an ordered mesophase with rectangular symmetry formed by two amphiphiles, sodium decyl sulfate and decanol, in presence of water, is investigated by neutron scattering. Our results show that the two amphiphiles are not uniformly distributed within the aggregate. Their relative concentration is modulated according to the local interfacial curvature of the aggregate. Such a concentration/curvature relationship may be an important point to understand the shape of the aggregates and therefore the polymorphism of lyotropic liquid crystals.
\end{abstract}

\section{Introduction.}

The polymorphism of the organization of amphiphilic molecules such as soaps, detergents or lipids, in presence of water, is now well described [1]. In the concentrated range of the phase diagrams the molecules can build structures which are periodic along one, two or three dimensions [2]. In this Letter, we shall focus our attention on the 2D and 1D ordered phases of the ternary system sodium decyl sulfate (SdS)/1-decanol/water at $23^{\circ} \mathrm{C}$ and the transformations from one into the other. It can be seen in the phase diagram of this system [3] that the replacement of SdS by decanol in the binary SdS/water hexagonal phase induces the sequence of phase transformations shown in figure 1. In this sequence the 2D hexagonal phase $\left(\mathrm{H}_{\alpha}\right)$, where the molecules aggregate into infinite cylinders with circular section, transforms into a 2D rectangular phase $\left(\mathbf{R}_{\alpha}\right)$, where the molecules build infinite cylinders with non-circular section (infinite ribbons), then into a 1D lamellar phase $\left(\mathrm{L}_{\alpha}\right)$, where the molecules are arranged in infinite lamellae. Thus,

$\left(^{+}\right)$Laboratoire associé au CNRS (LA no 2). 


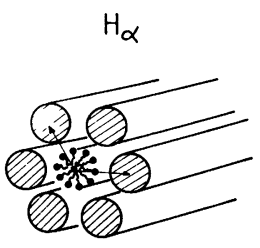

(a)

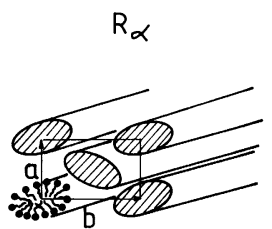

(b)

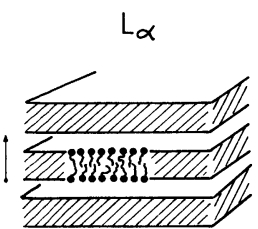

(c)

Fig. 1. - Schematic representation of the lyotropic mesophases discussed in the text. Composition of the phases (mole percent) : (a) $\mathrm{H}_{\alpha}$ (hexagonal phase) SdS : 7.1\%, decanol :0\%, $\mathrm{H}_{2} \mathrm{O}: 92.9 \%, T: 23{ }^{\circ} \mathrm{C}$. (b) $\mathrm{R}_{\alpha}$ (rectangular phase; space group pgg) SdS : 6.2\%, decanol :1.4\%; $\mathrm{H}_{2} \mathrm{O}: 92.4 \% ; T: 23{ }^{\circ} \mathrm{C}$. (c) $\mathrm{L}_{\alpha}$ (lamellar phase) SdS : $5.6 \%$, decanol : $2.4 \% ; \mathrm{H}_{2} \mathrm{O}: 92.0 \% ; T: 23{ }^{\circ} \mathrm{C}$.

the introduction of decanol into the binary $\mathrm{SdS} /$ water mixture appears as a powerful factor of polymorphism, the more so as the rectangular and lamellar phases do not exist in the binary $\mathrm{SdS} /$ water phase diagram at this temperature. We decided therefore to investigate more closely the role of decanol as it might provide a clue to the understanding of some mechanisms governing the shape and the organization of the aggregates.

Going back to figure 1, one can see that the phase transformations due to the addition of decanol are to be associated with a decrease of the interfacial curvature of the aggregates, from that of a cylinder to that of a lamella. The presence of decanol would therefore make flat interfaces more favourable. This implies that in a rectangular phase, whose ribbon-like aggregates exhibit non uniform interfacial curvature with highly curved edges and a rather flat body, the two amphiphiles might be heterogeneously distributed within an aggregate. The local concentration of decanol would be higher in the body than along the edges where SdS molecules would be preferentially located. We have explored this aspect of the internal structure of an aggregate by neutron scattering.

Before presenting and discussing our results we shall describe the characteristics of the rectangular phase studied and give a brief description of the experimental method.

\section{Structure of the rectangular phase.}

The sample selected for neutron scattering experiments has the following composition : $6.2 \%$ $\mathrm{SdS} / 1.4 \%$ decanol $/ 92.4 \%$ water (mole percent). At $23{ }^{\circ} \mathrm{C}$ this ternary mixture is monophasic, viscous and birefringent. The texture of the phase, observed under standard conditions with a polarizing microscope, resembles those repertoried for the so-called middle phases made of infinite cylinders organized in a 2D lattice, most often of hexagonal symmetry $[1,4]$. The structure of the phase was obtained by X-ray diffraction. The X-ray powder patterns show a set of five narrow Bragg reflexions in the small angle region which were indexed according to a bidimensional rectangular lattice of space group pgg. This implies that the $2 \mathrm{D}$ lattice with rectangular unit cell contains two cylindrical aggregates per unit cell whose cross section in the plane of the unit cell is not circular, the aggregates being depicted as infinite ribbons. The parameters of the rectangular unit cell are : $a=38 \AA$ and $b=106 \AA$; the dimensions of the ribbon cross section, thickness $\sim 19 \AA$ and width $\sim 52 \AA$, were estimated following a procedure already described in reference [3].

\section{Neutron scattering [5].}

Coherent scattering of neutrons is known to be much stronger for deuterons than it is for protons due to a large difference between their scattering lengths. This provides a convenient way to study the distribution of an amphiphilic species within an aggregate by deuterating its aliphatic 
part while that of the other species is kept protonated. If the distribution of the species within the aggregate is uniform, the aggregate will have a uniform scattering length density corresponding to the average value of the scattering length densities of the deuterated and protonated aliphatic chains. If, on the other hand, the distribution of the species is not uniform, the scattering length density of the aggregate will be modulated according to the distribution of the molecules, the amplitude of this modulation being $($ limited to the difference between the scattering length densities of the deuterated and protonated chains. The form factor of the aggregates will therefore be different in the two cases, i.e. the intensities of the Bragg reflexions of the rectangular lattice should be modulated differently. We propose here to look for an eventual inhomogeneous distribution of the amphiphiles by comparing the spectrum obtained with aggregates where both amphiphiles are protonated, a reference case where the scattering length density of the aggregate cannot be but homogeneous, with the spectrum obtained with aggregates where one type of amphiphilic molecule is deuterated, which contains the information relative to the distribution of the amphiphiles. If both spectra are similar, the distribution is homogeneous; if not, it is inhomogeneous.

For the sake of clarity we have developed this presentation of the method considering that the methylene and methyl groups of the paraffinic chains are the only scattering centres of an aggregate. However this is strictly speaking not correct since the polar heads of the amphiphilic molecules also contribute to the scattering with scattering length densities different from those of the methylene and methyl groups. The interfacial region, where the polar heads are localized, therefore constitutes another heterogeneity which might be the source of phenomena similar to the one discussed above. Another set of neutron scattering experiments, a contrast variation study of aggregates with protonated amphiphiles which we shall not detail here, shows that the contribution of the polar heads is small. However, it is difficult to estimate this contribution quantitatively in the absence of a model. Fortunately it was possible to get rid of it by studying the aggregates in a solvent whose scattering length density matches those of the polar heads. Indeed, if the solvent is a mixture of $26 \% \mathrm{H}_{2} \mathrm{O}$ and $74 \% \mathrm{D}_{2} \mathrm{O}$, its average scattering length density is $4.5 \mathrm{~cm}^{-2} \times 10^{10}$ while that of the $\mathrm{OSO}_{3} \mathrm{Na}$ group is $4.3 \mathrm{~cm}^{-2} \times 10^{10}$ and that of the hydroxyl group is $4.5 \mathrm{~cm}^{-2} \times 10^{10}$, taking into account the isotopic exchange ratio of its proton with the deuterons of $\mathrm{D}_{2} \mathrm{O}$ in the solvent [5].

For these experiments we prepared samples of the rectangular phase with the same molar composition but different states of deuteration. They were made either of protonated SdS and decanol or of protonated SdS and perdeuterated decanol in the above mixture of light and heavy water. We controlled by X-ray scattering measurements that the structure and structural parameters of the samples are independent of the percentages of deuterated molecules. The spectra were obtained with spectrometer $\mathrm{D}_{1} \mathrm{~B}$ of Institut Laue-Langevin (Grenoble). The spectrometer was equipped with a linear multidetector and we worked in the following $q$ range, $0.05 \AA^{-1}$ to $1 \AA^{-1}$. The polycrystalline samples were contained in quartz cells $1 \mathrm{~mm}$ thick mounted in a thermostated sample holder; the experiments were carried out at $23^{\circ} \mathrm{C}$. The data were normalized for unit incident beam flux. The spectra were corrected for background, absorption and detector efficiency. The width of the Bragg peaks is due to the instrumental resolution which also accounts for the fact that the higher orders, with low signal to noise ratio, are not always observed in these experiments.

\section{Experimental results and discussion.}

The spectrum obtained with aggregates containing protonated SdS and decanol is shown in figure $2 \mathrm{a}$; the spectrum obtained with aggregates containing protonated SdS and perdeuterated decanol is shown in figure $2 \mathrm{~b}$. Both spectra show three Bragg reflexions corresponding to the three first orders of the X-ray spectrum. If we compare the intensities of the orders of spectrum 2a (sample with fully protonated aggregates) with those of the corresponding orders of spectrum 


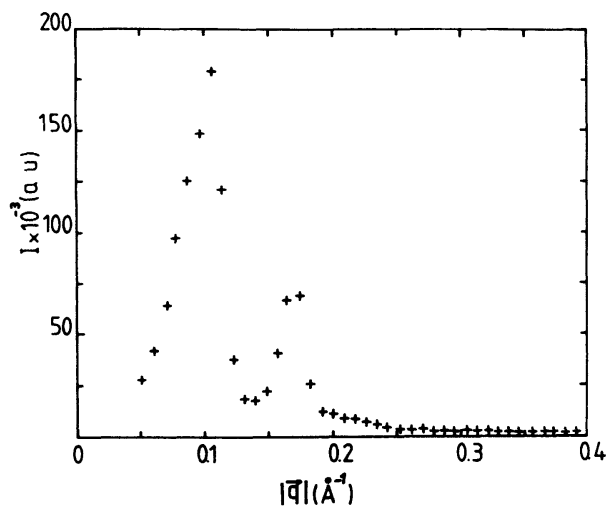

(a)

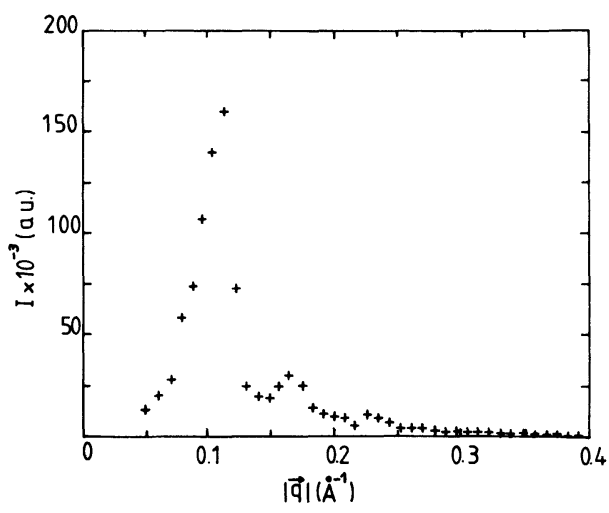

(b)

Fig. 2. - Neutron scattering spectra of the rectangular phase. Sample composition : (a) protonated SdS and protonated decanol in $74 \% \mathrm{D}_{2} \mathrm{O}$. (b) protonated SdS and perdeuterated decanol in $74 \% \mathrm{D}_{2} \mathrm{O}$. Solvent composition in mole percent.

$2 \mathrm{~b}$ (sample with partially deuterated aggregates) we observe that the first orders are in the ratio 1.1 , the second orders in the ratio 2.4 and the third ones in the ratio 0.8 . The two spectra are therefore not similar and following the discussion in the preceding section, the form factor of the partially deuterated aggregate is different from that of the fully protonated one. We can therefore conclude that the distribution of the molecules within the aggregates is inhomogeneous. Another evidence for this conclusion is the fact that the intensities of the first orders are nearly equal. Indeed, in a more quantitative manner, if the molecules were uniformly distributed, the scattering length density of the aggregate with deuterated decanol would also be uniform $\left(\bar{\rho}=0.96 \mathrm{~cm}^{-2} \times 10^{10}\right.$ and $\left.\bar{\rho}-\rho_{\mathrm{s}}=-3.54 \mathrm{~cm}^{-2} \times 10^{10}\right)$ and the intensity should be twice smaller than in the case of the sample with fully protonated aggregates $\left(\rho=-0.41 \mathrm{~cm}^{-2} \times\right.$ $10^{10}$ and $\rho-\rho_{\mathrm{s}}=-4.91 \mathrm{~cm}^{-2} \times 10^{10}$ ).

The next step would consist in determining which molecule, $\mathrm{SdS}$ or decanol is preferentially located in the flattened parts of the aggregates. The analysis of the contrast variation study which would provide this kind of information requires the development of a model which is in progress. Nevertheless, going back to the sequence sketched in figure 1 where it appears that the addition of decanol lowers the mean interfacial curvature, we may infer that decanol molecules should preferentially be located in the flattened core of the ribbons and, consequently, that the concentration of SdS should be higher along their edges [6].

Finally, if the segregation we observed is to be ascribed to the different nature of the polar heads of amphiphiles which have the same chain length, it is interesting to notice that a similar effect may also be observed when amphiphiles with the same polar head but different chain lengths are mixed together [7].

\section{Conclusion.}

A mesophase of the SdS/1-decanol/water system has been studied. The symmetry of the phase is rectangular, its aggregates are infinite rods with non circular section, i.e. the curvature of the amphiphile-water interface is inhomogeneous. Our experimental data demonstrate that the distribution of the amphiphilic molecules within the aggregates is inhomogeneous as well. From this we may infer that the non uniformity of the interfacial curvature is correlated with that of the relative concentration of the amphiphiles. Some hypothesis about the mechanisms 
which may govern the formation of ribbon-like aggregates and their arrangement in a rectangular lattice can thus be developed on the basis of these observations.

Let us first consider only the isolated aggregate, or intra-aggregate properties. Obviously, decanol contributes to a change of the interfacial curvature and the inhomogeneous curvature of the ribbon-like aggregates of the $R_{\alpha}$ phase integrated over the whole surface of the ribbon has a value intermediate between $1 / R$ (cylinders with radius $R$ ) and $\sim 0$ (lamellae with $R \rightarrow \infty$ ). If such an intermediate value were to be achieved in an homogeneous way the radius of the cylinder with circular section in the $\mathrm{H}_{\alpha}$ phase should increase from its $R$ value. As this radius $R$ is roughly equal to the length of an amphiphilic molecule this increase would require the formation of a hole in the paraffinic core of the aggregates with a new interface, and therefore an extra energy cost. The ribbon-like structure with segregated molecules may be a solution chosen by the system to accomodate this new energy term while the curvature changes.

Let us now consider the packing of the aggregates, or interaggregates properties. This packing is governed by the interaction between the aggregates which contains a repulsive electrostatic term. The section of the aggregate being anisotropic the electric potential has a quadrupolar component and the non uniform distribution of the amphiphilic molecules, which induces an inhomogeneous surface charge density, certainly contributes to its magnitude. This might account for the transformation of the pgg rectangular phase into the $\mathrm{cmm}$ phase described in [3], as the decanol concentration decreases.

\section{Acknowledgments.}

The help of M. Rawiso on the spectrometer of I.L.L. is gratefully acknowledged. This work was partially supported by PIRSEM (CNRS).

\section{References}

[1] Ekwall, P., Liq. Cryst. 1 (edited by G. H. Brown, Academic Press) 1975, p. 1.

[2] Luzzati, V., Biol. Memb. 1 (edited by D. Chapman, Academic Press) 1965, p. 71. Skoulios, A., Adv. Colloid Interface Sci. 1 (1967) 79.

[3] Hendrikx, Y. and Charvolin, J., J. Physique 42 (1981) 1427. The decanol concentration of the rectangular phase in this reference is 1.15 mole percent. Its lattice has the symmetry of space group $\mathrm{cmm}$ whereas the rectangular phase we are dealing with in the present paper has the symmetry of space group pgg. The evolution of one space group to the other, as a function of the decanol concentration will be analysed in a forthcoming paper.

[4] Rosevear, F. B., J. Soc. Cosmet. Chem. 19 (1968) 581.

[5] JACROT, B., Rep. Prog. Phys. 39 (1976) 911. We recall that the scattering length densities used in this work are, in $\mathrm{cm}^{-2} \times 10^{10}: \mathrm{CD}_{2}: 7.41 ; \mathrm{CH}_{2}:-0.31 ; \mathrm{CD}_{3}: 4.93 ; \mathrm{CH}_{3}:-0.85 ; \mathrm{H}_{2} \mathrm{O}:-0.56$; $\mathrm{D}_{2} \mathrm{O}: 6.40 ; \mathrm{OSO}_{3} \mathrm{Na}: 4.33 ; \mathrm{OH}: 0.98 ; \mathrm{OD}: 5.70$.

[6] A similar segregation was recently observed in finite disk-like aggregates of a lyotropic nematic phase of the ternary system, potassium laurate/1-decanol/water : Hendrikx, Y., ChARvolin, J. and Rawiso, M., J. Colloid Interface Sci. 100 (1984) 597. Since this phase has no long range translational order the discrimination between structure and form factors was not as straightforward as in the present case.

[7] Chidichimo, G., Golemme, A. and Doane, J. W., private communication of a preprint before publication. 